\title{
Effects of Smoking, and Drinking on Serum Gamma-Glutamyl Transferase Levels Using Physical Examination Data: A Cross-Sectional Study in Northwest China
}

This article was published in the following Dove Press journal:

International Journal of General Medicine

\section{Zhuo Zhang' \\ Lu Ma ${ }^{2}$ \\ Hui Geng ${ }^{3}$ \\ Ying Bian'}

'State Key Laboratory of Quality Research in Chinese Medicine, Institute of Chinese Medical Sciences, University of Macau, Taipa, Macau, People's Republic of China; ${ }^{2}$ Xi'an Shiyou University, Xi'an, Shaanxi, People's Republic of China; ${ }^{3}$ Physical Examination Center, The First Affiliated Hospital of Xi'an Jiaotong University, Xi'an, Shaanxi, People's Republic of China
Correspondence: Ying Bian Institute of Chinese Medical Sciences, University of Macau, Avenida Da

Universidade, Room 2055, N22 Building, Taipa, Macau SAR, People's Republic of China

Tel +86853-66548926

Email bianyingum@163.com
Purpose: Although drinking and smoking have been associated with gamma-glutamyl transferase (GGT) levels, studies on the effects of smoking and drinking on GGT levels are scarce. The objective of this study was to assess the individual and combined effects of smoking, drinking on GGT levels in the Shaanxi province of Northwest China.

Participants and Methods: A questionnaire survey was conducted in a population that underwent health examination at the First Affiliated Hospital of Xi' an Jiaotong University and included employees of enterprises or public institutions. The survey was used to collect the baseline characteristics, smoking status, and drinking status of the participants. This information was collected from January 2019 to December 2019. Data related to the physical examinations were exported using the hospital information system (HIS). A linear regression model was employed to explore the effects of smoking and drinking on GGT levels. The restricted cubic spline model was applied to assess the dose-response relationship between amount of smoking, alcohol consumption and GGT levels.

Results: A total of 10,177 participants were included in the study. Linear regression indicated that smoking $(\beta=3.37,95 \% \mathrm{CI}: 2.57-4.17)$ and drinking $(\beta=5.55 \mathrm{~L}, 95 \% \mathrm{CI}$ : $4.40-6.71)$ individually, and collectively $(\beta=9.30,95 \% \mathrm{CI}: 7.83-10.76)$ had a positive effect on GGT levels. The restricted cubic spline presented a linear dose-response relationship between the amount of daily smoking and GGT levels ( $\mathrm{P}$ for non-linearity $=0.148, \mathrm{P}$ for overall association $<0.001, \mathrm{OR}=2.49,95 \% \mathrm{CI}$ : $1.27-4.90)$, and that between weekly alcohol consumption and GGT levels ( $\mathrm{P}$ for non-linearity $=0.231$, $\mathrm{P}$ for overall association $<0.001$, $\mathrm{OR}=4.79$, 95\% CI: 1.72-13.32). In case of females, stratified analysis showed that in comparison to the reference group, only current drinkers had a significant effect on GGT levels (OR=3.37, 95\% CI: 0.19-6.55).

Conclusion: Smoking and drinking have a dose-dependent and a synergistic effect on GGT levels. They should be controlled concurrently, especially among males.

Keywords: gamma-glutamyl transferase, smoking, drinking, restricted cubic spline

\section{Introduction}

Gamma-glutamyl transferase (GGT) is an enzyme that exists in various tissues, the most notable one being the liver. Moreover, it is used as a diagnostic marker in medicine. ${ }^{1}$ Particularly, GGT can be used as an early stage marker of oxidative stress-related events that are caused by alcohol consumption and current smoking. ${ }^{2}$ 
GGT has been of substantial interest for its predictive potential for a variety of clinical outcomes such as metabolic syndrome, cardiovascular disease, progression of cancer, diabetes, and chronic obstructive pulmonary disease in both generally healthy and patient cohorts. ${ }^{3-7}$ Therefore, a better understanding of the factors determining individuals' GGT levels has been a matter of public health concern. Excessive drinking is estimated to be the fourth leading preventable cause of death in the United States. ${ }^{8}$ A large body of evidence supports the idea that damage from alcohol-related cirrhosis is not reversible and causes fatal liver failure. ${ }^{9}$ Among all other routinely assessed liver enzymes, GGT is the most sensitive measure of alcohol consumption. Previous studies have indicated a significant dose-response relationship between alcohol consumption and GGT levels. ${ }^{10,11}$ In addition, studies have shown that a variety of toxins in cigarettes can change the enzymatic and inflammatory pathways in liver physiology, leading to liver dysfunction and disease. ${ }^{11}$ Moreover, recent studies have shown associations between smoking and GGT. ${ }^{10,12,13}$

So far, our knowledge of the combined effects of drinking and cigarette smoking on the status of the liver biomarker (GGT) is limited in the Chinese population, and a better understanding of the role of disease risk factors and the inter-individual variation of GGT provides a rationale for examining this topic. We argue that studying the individual and combined effects of smoking and drinking is important because it advances our understanding of the harmful effects of nicotine and alcohol use on a large number of comorbidities in humans.

The aim of this study was to examine the individual, and combined effects of drinking and smoking on serum GGT levels using physical examination data from a large tertiary hospital in Northwest China. The data included detailed records on smoking, drinking, self-reported health status, sleep conditions, and physical activity. In our study, we tried to use a restricted cubic spline model to explore the dose-response relationship between smoking, drinking, and serum GGT levels. We proposed the following hypotheses:

Hypothesis 1: Smoking amount has a dose-dependent effect on GGT levels.

Hypothesis 2: Drinking amount has a dose-dependent effect on GGT levels.

Hypothesis 3: Smoking and drinking have a synergistic effect on GGT levels.
Hypothesis 4: The association of smoking and drinking with GGT varies by gender.

\section{Participants and Methods Study Design and Participants}

This analysis is based on data from the routine occupational health examinations (from January to December 2019) of participants aged 18 years or older, who underwent comprehensive annual examinations at the First Affiliated Hospital of Xi'an Jiaotong University in Shaanxi, China. Most participants who were included in the study were employees at enterprises and public institutions. Compared with the Shaanxi Statistics Yearbook, the population of health examinations has a larger proportion of highly educated people that are over 25 years of age.

\section{Data Collection}

The health examinations were conducted by experienced nurses in a standardized manner, and after obtaining informed consent. The study population fasted for at least $8 \mathrm{~h}$ prior to the examinations to reduce any potential physiological confounding factors. Uniformly trained nurses administered a standard questionnaire on exposure to smoking and drinking. Physical measurements (such as height and weight) were made, and blood samples were collected to prepare serum for the analysis of biochemical and hematological variables.

A total of 11,093 health examination records were collected. After excluding subjects who had incomplete key variables or outcomes (820 individuals without GGT examination, 21 individuals with missing information on smoking or drinking), and participants with liver diseases such as viral hepatitis or liver cirrhosis that can cause abnormalities in various liver enzyme indexes (84 individuals with positive hepatitis B surface), 10,177 subjects remained in this study.

Nurses with a medical background and fieldwork experience served as investigators. Before the investigation, uniform training and on-site internships were performed for all investigators. After qualifying their respective internships, the investigators participated in the present study. The investigation handbook was compiled, and all procedures were carried out in accordance to the manual. After completing a paper questionnaire, to ensure the accuracy of the data, the supervisors reviewed the questionnaires and checked for logistical errors and missing values. If there were any problems with the questionnaire, the investigator would revise it in time. 


\section{Ascertainment of Study Variables}

The main outcome ie, serum GGT levels, were measured on a Beckman Coulter Chemistry analyzer (AU5431 Chemistry analyzer, Beckman Coulter, USA) after verifying the performance standards of analytical instruments and tools, following the recommendations of the Clinical and Laboratory Standards Institute. ${ }^{14}$

Smoking and drinking were considered as exposure variables. Smokers were categorized as non-smoker, current smoker, or ex-smoker. Non-smokers represented individuals who did not smoke currently and those that smoked $<100$ cigarettes during their lifetime. Current smokers were defined as those participants who smoked daily, on most days, or had quit smoking for less than six months. In particular, regular smokers who had stopped smoking $\geq 6$ months before recruitment due to illness were included in the current smoking group to avoid bias. Ex-smokers were those who did not smoke or had been smoking only occasionally for more than six months, but in the past, had smoked on most days or daily. The amount of tobacco smoked ( $\mathrm{g} /$ day, $1 \mathrm{~g}=1$ cigarette) by each smoker was calculated according to the smoker's self-reported data on the type and amount of tobacco smoked. It was assumed that the quantity of tobacco smoked by the participants was $1 \mathrm{~g}$ per factory cigarette and $2 \mathrm{~g}$ per cigar, whereas that in the case of hand-rolled cigarettes and pipes was considered as $50 \mathrm{~g}$ per month.

Participants were asked to report their drinking frequency during the previous 12 months (never or almost never; occasionally; usually at least once a week). The subjects were then classified into three categories: abstainers (who had never or almost never drunk in the past 12 months), occasional drinkers (who drank occasionally), and weekly drinkers (who usually drank at least once a week). Participants would be asked further questions if they consumed alcohol at least once a week: how many days in a typical week they drank ((1-2, 3-5 or 6-7 days per week), the type (beer, wine, or spirits), the amount of each type drunk (beer, described by number of small $(250 \mathrm{~mL})$ or large $(640 \mathrm{~mL})$ bottles; wines and spirits, number of liang (50 g)) on a typical drinking week. Ethanol (in g) consumed in a typical drinking week was then calculated by referring to the literature for determining the alcohol content by volume $(\mathrm{v} / \mathrm{v})$, of different varieties typically found in China. ${ }^{15}$

\section{Assessment of Covariates}

Adjustments were made for the covariates that may influence the association between smoking, drinking and
GGT: sex (male, female), age ( $\leq 35,35-65$ y), education (senior high school and below, college, postgraduate and above), marital status (married, single), physical activities (almost no participation,1-3 per month, $\geq 1$ /week), annual household income $(<50,000,500,000$, and $\geq 100,000$ yuan), self-reported health (best, better, good, normal, bad), sleeping problem (no, yes), and body mass index (BMI) at recruitment (in 4 categories: $<18.5$ was underweight; 18.5-23.9 was normal weight; 24-27.9 was overweight, and $\geq 28.0$ was obese). BMI was calculated as weight in kilograms divided by the square of standing height in meters $(\mathrm{kg} / \mathrm{m} 2)$. With participants wearing light, weight was measured by a body composition analyser (TANITA-TBF-300GS; Tanita Corporation, Tokyo, Japan). A calibrated stadiometer was used to obtain the standing height.

\section{Statistical Analysis}

The data of questionnaire were duplicate entered into the database designed by EpiData 3.1 and the data related to physical examination were exported through the hospital information system (HIS), data cleaning was performed using STATA 15.1. Counts and proportions were used to describe the categorical variables. Mean and standard deviation (SD) were used to describe the continuous variables, and $t$ test or one-way anova were used to examine difference between groups.

A linear regression model was used to explore the association between smoking, drinking, and GGT levels. The data was adjusted for no variables in model I, sex and age in model II, and sex, age, marital status, annual household income, BMI, physical activities, self-reported health, and sleeping problems in model III. The restricted cubic spline model was employed to assess the dose-response relationship between the amount of smoking, alcohol consumption, and GGT levels in smokers and drinkers. To make the effect more significant, GGT was used as a categorical variable, divided by the upper and lower quantiles in this model. In addition, we conducted a stratified analysis based on gender. To rule out potential confusion due to the classification standard, we conducted sensitivity analyses by shifting participants, who quit smoking due to illness, from the current smoking group to the quit smoking group.

STATA 15.1 (StataCorp LP) was used to perform the statistical analyses, and a two-tailed $\mathrm{P}<0.05$, was considered statistically significant. 


\section{Ethics Statement}

The present research was based on the Declaration of Helsinki, and the first affiliated hospital of Xi'an Jiaotong University had approved this research (No. XJTU1AF2020LSK-091). The investigators acquired written informed consent from all participants before investigation.

\section{Results}

\section{The Characteristics of the Participants}

A total of 10,177 participants were included in the study. The average age was $41.33 \pm 13.68 \mathrm{y}, 52 \%$ were males, and $62 \%$ were married. Current smokers and current drinkers accounted for $20.91 \%$ and $8.04 \%$ of the participants, respectively. Most patients had college level of education (53.91\%) and a normal BMI (50.4\%). Details about the characteristics of these participants are presented in Table 1. The average level of GGT was $24.23 \pm 16.02 \mathrm{U} / \mathrm{L}$. There was a significant $(\mathrm{P}<0.05)$ difference in GGT levels across sex, age, education, marital status, BMI, self-reported health, smoking, and alcohol consumption.

\section{The Association Between Smoking,} Alcohol Consumption, and GGT Levels

With all confounding factors adjusted, the linear regression showed that GGT levels in current smokers had an average increase of $3.37 \mathrm{U} / \mathrm{L}$ (95\% CI: 2.57-4.17) in comparison to non-smokers, whereas current drinkers had an average increase of $5.55 \mathrm{U} / \mathrm{L}$ (95\% CI: 4.40-6.71) compared to abstainers. The differences (Table 2) were statistically significant $(\mathrm{P}<0.001)$. Table 3 displays the effect of the interaction between smoking, alcohol consumption, and GGT levels. Compared to non-smokers and abstainers, current smokers and current drinkers had an average increase of $9.30 \mathrm{U} / \mathrm{L}$ (95\% CI: 7.83-10.76, $\mathrm{P}<0.05)$ in their GGT levels.

\section{The Dose-Response Relationship} Between the Amount of Smoking, Alcohol Consumption, and GGT

The restricted cubic spline presented a linear doseresponse between the amount of daily smoking and GGT levels ( $\mathrm{P}$ for non-linearity association $=0.148$ and $\mathrm{P}$ for overall association $<0.001)$. An increase in every 10 cigarettes every week (Figure 1) was associated with a $37 \%$ increase in GGT level ( $\mathrm{OR}=2.49$, 95\% CI: 1.27-4.90).
Figure 2 shows a linear dose-response between the amount of weekly drinking and GGT levels ( $\mathrm{P}$ for non-linearity association $=0.231$ and $\mathrm{P}$ for overall association $<0.001)$. A $100 \mathrm{~g}$ increase in weekly drinking was associated with a $63 \%$ increase in GGT levels (OR=4.79, 95\% CI: 1.72-13.32).

\section{Subgroup Analysis}

We further conducted a stratified analysis based on sex. The effect of smoking and drinking on GGT levels among men did not change after stratification. Whereas in case of females, compared to the reference group, only current drinkers exhibited a significant effect $(\beta=3.37,95 \% \mathrm{CI}$ : 0.19-6.55) on GGT levels (Table 4). Moreover, the doseresponse relationship and interaction between amount of smoking, alcohol consumption, and GGT levels did not change after stratification in males. In case of females, no interaction between smoking, drinking, and GGT levels was found, and the dose-response relationship did not change significantly due to the low amount of smoking and drinking.

\section{Sensitivity Analysis}

Additional sensitivity analysis was performed by separating participants who had quit smoking due to an illness, from those who belonged to the current smoking group $(n=40)$. However, this did not change the results (data not shown).

\section{Discussion}

GGT is increasingly recognized as a biomarker for the risk of developing cardiovascular and metabolic syndromes. ${ }^{16,17}$ Consequently, expanding our understanding of the relationships between the main exposures in our study (drinking and smoking) and GGT levels has become increasingly important. In the present study, we demonstrate a gradual increase in GGT levels with increasing levels of daily smoking and weekly drinking. Furthermore, there was a synergistic interaction between smoking and drinking with respect to GGT levels. Since these two risk factors are relatively prevalent in the general population, preventive health measures should be strengthened at all possible levels, and individuals should be encouraged to avoid the use of these intoxicants in combination, thereby reducing mortality and the possibility of developing various chronic diseases.

Consistent with some of the previous reports, ${ }^{13,18}$ we found a positive association between smoking and GGT levels that can be explained by oxidative stress and 
Table I Characteristics of the Participants

\begin{tabular}{|c|c|c|}
\hline Variables & $\mathbf{N}(\%)$ & GGT (U/L)* \\
\hline \multicolumn{3}{|l|}{ Gender } \\
\hline Male & $5347(52.54)$ & $30.03(17.04)^{\S}$ \\
\hline Female & $4830(47.46)$ & 17.81 (11.89) \\
\hline \multicolumn{3}{|l|}{ Age (year) } \\
\hline$\leq 35$ & $4283(42.09)$ & $22.99(16.23)^{\S}$ \\
\hline $35 \sim$ & $5219(51.28)$ & $25.22(15.96)$ \\
\hline$\geq 65$ & $675(6.63)$ & $24.5 I(14.57)$ \\
\hline \multicolumn{3}{|l|}{ Education } \\
\hline Senior high school and below & $3492(34.31)$ & $25.09(15.84)^{\S}$ \\
\hline College & $5486(53.91)$ & $24.00(16.19)$ \\
\hline Postgraduate and above & $1199(11.78)$ & $22.83(15.65)$ \\
\hline \multicolumn{3}{|l|}{ Marital status } \\
\hline Married & $8264(81.20)$ & $24.77(16.31)^{\S}$ \\
\hline Single & $1913(18.80)$ & 21.91 (14.49) \\
\hline \multicolumn{3}{|l|}{ Annual household Income (yuan) } \\
\hline$<50,000$ & $2616(25.7 I)$ & $24.31(15.66)$ \\
\hline $500,000 \sim$ & 3601 (35.38) & $23.94(15.47)$ \\
\hline$\geq 100,000$ & $3960(38.91)$ & $24.45(16.74)$ \\
\hline \multicolumn{3}{|l|}{ BMI (kg/m2) } \\
\hline$<18.5$ & $376(3.69)$ & $15.49(9.10)^{\S}$ \\
\hline $18.5 \sim 23.9$ & $5180(50.90)$ & $19.34(12.55)$ \\
\hline $24 \sim 27.9$ & $3473(34.13)$ & $28.62(16.73)$ \\
\hline$\geq 28$ & II 48 (II.28) & $35.93(19.07)$ \\
\hline \multicolumn{3}{|l|}{ Physical activities } \\
\hline Almost no participation & $2923(28.72)$ & $24.29(16.66)$ \\
\hline $\mathrm{I} \sim 3 /$ month & $2619(25.73)$ & $24.49(16.33)$ \\
\hline$\geq \mathrm{I} /$ week & $4635(45.54)$ & $24.06(15.43)$ \\
\hline \multicolumn{3}{|l|}{ Self-reported health } \\
\hline Best & $503(4.94)$ & $22.66(15.23)^{\S}$ \\
\hline Better & $2282(22.42)$ & $23.49(14.92)$ \\
\hline Good & $3300(32.43)$ & 23.99 (15.87) \\
\hline Normal & 3826 (37.59) & $24.90(16.66)$ \\
\hline $\mathrm{Bad}$ & $266(2.61)$ & $27.19(18.38)$ \\
\hline \multicolumn{3}{|l|}{ Sleeping problem } \\
\hline No & $6352(62.42)$ & $24.04(15.80)$ \\
\hline Yes & $3825(37.58)$ & $24.57(16.39)$ \\
\hline \multicolumn{3}{|l|}{ Tobacco use } \\
\hline Nonsmoker & 7751 (76.16) & $21.72(14.48)^{\S}$ \\
\hline Ex-smoker & $298(2.93)$ & $29.21(15.84)$ \\
\hline Current smoker & $2128(20.91)$ & $32.70(18.20)$ \\
\hline \multicolumn{3}{|l|}{ Alcohol use } \\
\hline Abstainer & 3915 (38.47) & $20.68(13.79)^{\S}$ \\
\hline Occasional drinker & 5444 (53.49) & $25.17(16.23)$ \\
\hline Current drinker & $818(8.04)$ & $35.03(18.68)$ \\
\hline
\end{tabular}

Notes: *Values were expressed as mean (SD) for the continuous variable. ${ }^{\S} \mathrm{p}<0.05$.

Abbreviations: GGT, gamma-glutamyl transferase; BMI, body mass index. 
Table 2 The Influence of Smoking and Alcohol Consumption on GGT Level

\begin{tabular}{|c|c|c|c|c|c|c|}
\hline & \multicolumn{2}{|c|}{ Model I } & \multicolumn{2}{|c|}{ Model II* } & \multicolumn{2}{|c|}{ Model III§ } \\
\hline & $\beta(95 \% \mathrm{Cl})$ & $\mathbf{P}$ & $\beta(95 \% \mathrm{Cl})$ & $\mathbf{P}$ & $\beta(95 \% \mathrm{Cl})$ & $\mathbf{P}$ \\
\hline \multicolumn{7}{|l|}{ Tobacco Use } \\
\hline Nonsmoker & Ref. & & Ref. & & Ref. & \\
\hline Ex-smoker & $5.72(3.95,7.49)$ & $<0.001$ & $0.29(-1.46,2.03)$ & 0.747 & $-0.10(-1.78,1.58)$ & 0.907 \\
\hline Current smoker & $8.87(8.10,9.64)$ & $<0.001$ & $3.99(3.17,4.8 I)$ & $<0.001$ & $3.37(2.57,4.17)$ & $<0.001$ \\
\hline \multicolumn{7}{|l|}{ Alcohol use } \\
\hline Abstainer & Ref. & & Ref. & & Ref. & \\
\hline Occasional drinker & $2.88(2.24,3.5 \mathrm{I})$ & $<0.001$ & $0.53(-0.12,1.18)$ & 0.111 & $0.44(-0.19,1.06)$ & 0.171 \\
\hline Current drinker & $9.75(8.54,10.96)$ & $<0.001$ & $5.99(4.80,7.19)$ & $<0.001$ & $5.55(4.40,6.7 I)$ & $<0.001$ \\
\hline
\end{tabular}

Notes: *Gender and age were adjusted. ${ }^{\S}$ All variables in model II plus education, marital status, annual household income, BMI, physical activities, self-reported health and sleeping problem were adjusted.

Table 3 The Interaction of Smoking and Alcohol Consumption on GGT Level

\begin{tabular}{|l|c|c|c|c|}
\hline \multicolumn{2}{|c|}{} & Model I & Model II* & Model III $^{\S}$ \\
\cline { 3 - 5 } & $\boldsymbol{\beta ( 9 5 \% ~ C l )}$ & $\boldsymbol{\beta ( 9 5 \% ~ C l )}$ & $\boldsymbol{\beta ( 9 5 \% ~ C l )}$ \\
\hline Smoking & $\begin{array}{c}\text { Alcohol } \\
\text { consumption }\end{array}$ & & & \\
\hline $\begin{array}{l}\text { Current } \\
\text { Smoker }\end{array}$ & $\begin{array}{c}\text { Current } \\
\text { drinker }\end{array}$ & $\begin{array}{c}17.93(19.30, \\
20.30)^{\#}\end{array}$ & $\begin{array}{c}10.15(8.63, \\
11.67)\end{array}$ & $\begin{array}{c}9.30(7.83, \\
10.76)^{\#}\end{array}$ \\
\hline
\end{tabular}

Notes: The reference group is nonsmoker and abstainer. ${ }^{*}$ Gender and age were adjusted. ${ }^{\S} \mathrm{All}$ variables in model II plus education, marital status, annual household income, BMI, physical activities, self-reported health and sleeping problem were adjusted. ${ }^{\#} \mathrm{P}<0.05$

inflammation. ${ }^{19}$ In contrast, a study by Breitling noted that smoking itself had no impact on GGT levels. ${ }^{20}$ Similar to previous findings, the present study also reports an excess of GGT in ex-smokers compared to non-smokers. ${ }^{21,22}$ This can be explained by the fact that quitting smoking leads to weight gain, and that GGT levels have been shown to be strongly influenced by weight. ${ }^{23}$ This is particularly seen in cases where health issues associated with GGT elevations have led smokers to quit smoking. It is well known that GGT plays an important role in antioxidant defense systems, ${ }^{24}$ and that oxidative stress plays a role in the development of obesity. ${ }^{23}$ However, the excess of GGT in ex-smokers was not significant in this study and was consistent with a previous study conducted among 15,000 German male workers. ${ }^{25}$ Further research would be needed to understand the deep-seated relationship between the two.

The restricted cubic spline indicates a dose-response relationship, where in agreement with previous studies, the mean GGT levels increase with alcohol consumption, ${ }^{26,27}$ and confirms that GGT induction begins when low-levels of alcohol are consumed. However, some analyses have shown other types of dose-response curves. For example, a study provided evidence that light to moderate drinking had protective effects and led to a dose-dependent decrease in GGT levels. ${ }^{28}$ Sanjiv Agarwal's survey showed both curvilinear and linear equations with respect to the GGT levels and alcohol consumption. ${ }^{29}$ In the present study, quantitative estimates calculated based on the association between the amount of smoking and GGT levels, or alcohol consumption and GGT levels are not non-linear. It may be possible that alcohol has different effects on health in different populations. Conversely, this may also be the result of insufficient statistical power caused by the fewer numbers of smokers or drinkers.

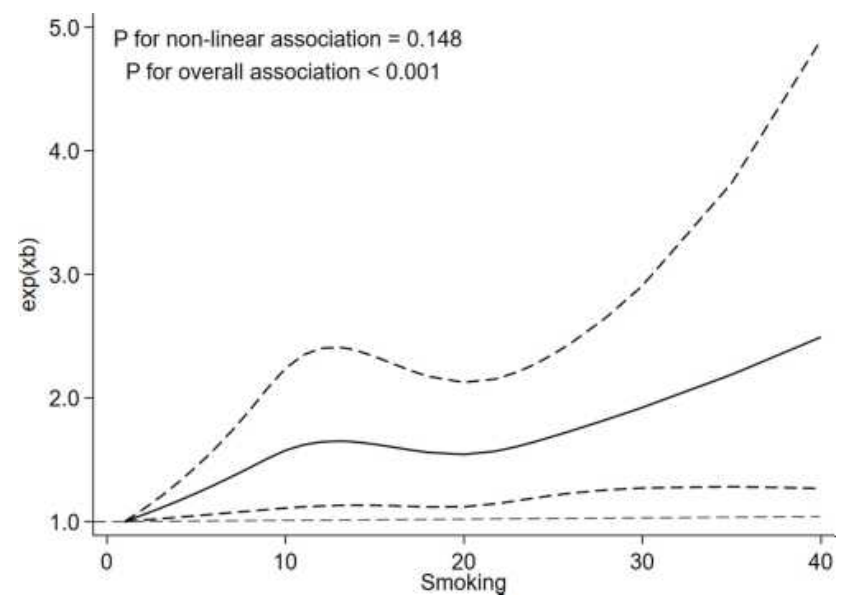

Figure I The dose-response relationship between smoking and GGT levels. Gender, age, education, marital status, annual household income, BMI, physical activities, self-reported health and sleeping problem were adjusted. Four knots (8, $10,20,25)$ were selected in the model. 


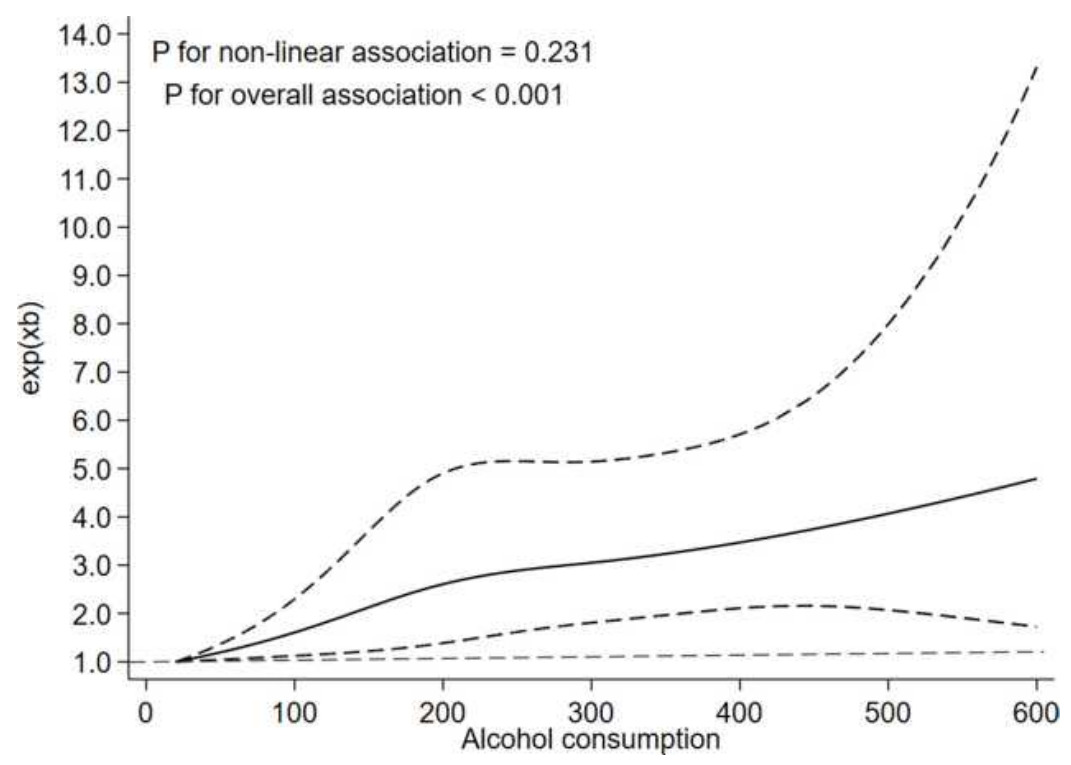

Figure 2 The dose-response relationship between alcohol consumption and GGT levels. Gender and age, education, marital status, annual household income, BMI, physical activities, self-reported health and sleeping problem were adjusted. Four knots $(95,185,300,500)$ were selected in the model.

Present observations show that smoking and drinking have a significant and a synergistic effect on the elevation of GGT levels, which agrees with previous studies. ${ }^{20,22,27}$ Additionally, drinking and smoking can together have hepatotoxic effects. In fact, another study proved that this hepatotoxic effect was more pronounced in men. ${ }^{20,30}$ This can be explained by the fact that oxidative stress plays a key role in the development of alcoholic liver disease. Smoking and drinking can aggravate and enhance oxidative stress in the liver, leading to more serious liver cell damage. Breitling hypothesized that both drinking and smoking could lead to the consumption of oxidative stress defense substances (such as glutathione) in different tissues, and that the two had an additive effect. ${ }^{20}$
To the best of our knowledge, previous studies were mostly conducted among men. ${ }^{12,31}$ Analysis by gender stratification revealed that the results remained unaltered among males, whereas in the case of females, only current drinkers had a significant effect on GGT levels. This may be because women smoked and drank less, ie, not enough to cause an increase in GGT levels. Alternatively, it may be explained by insufficient test efficiency due to the small number of women smokers and drinkers.

The advantages of this study include the large sample size, and a comprehensive assessment of exposure factors and biomarkers involved in the diagnosis of hepatobiliary diseases. Studies on the potential interaction between smoking, drinking, and GGT levels in the Chinese

Table 4 The Effect of Smoking and Alcohol Consumption on GGT Level by Gender*

\begin{tabular}{|c|c|c|c|c|}
\hline & \multicolumn{2}{|c|}{ Male } & \multicolumn{2}{|c|}{ Female } \\
\hline & $\beta(95 \% \mathrm{Cl})$ & $\mathbf{P}$ & $\beta(95 \% \mathrm{Cl})$ & $\mathbf{P}$ \\
\hline \multicolumn{5}{|l|}{ Tobacco Use } \\
\hline Nonsmoker & Ref. & & Ref. & \\
\hline Ex-smoker & $0.37(-1.55,2.30)$ & 0.703 & $0.02(-9.94,9.99)$ & 0.996 \\
\hline Current smoker & $3.4 \mathrm{I}(2.47,4.34)$ & $<0.001$ & I.23 (-2.42, 4.87) & 0.509 \\
\hline \multicolumn{5}{|l|}{ Alcohol use } \\
\hline Abstainer & Ref. & & Ref. & \\
\hline Occasional drinker & $1.14(0.04,2.25)$ & 0.043 & $-0.14(-0.8 \mathrm{I}, 0.52)$ & 0.669 \\
\hline Current drinker & $6.11(4.60,7.62)$ & $<0.001$ & $3.37(0.19,6.55)$ & 0.038 \\
\hline
\end{tabular}

Notes: *age, education, marital status, annual household income, BMI, physical activities, self-reported health and sleeping problem were adjusted. 
population are scarce. The difference between our study and previous studies is that we used a spline regression model while taking the amount of smoking and alcohol consumption as continuous variables, and in a Chinese population to explore the dose-response relationship between the amount of smoking, alcohol consumption, and GGT levels. In addition, the study participants belonged to the population that underwent hospital physical examination, and the results obtained are "real-world evidence," therefore more pertinent to the general population. Finally, this study gradually incorporated the covariates into the regression model and found that the relationship between smoking, drinking, and GGT levels in several models was relatively stable Therefore, the results obtained in this study can be considered effective.

Nevertheless, our study has some limitations. First, alcohol consumption was self-reported by the respondents, and is therefore prone to bias and might have led to underestimation of the true dose-response association. Second, as this study is a cross-sectional study, we were unable to establish any cause and effect associations. Therefore, it is necessary to develop a cohort study with a large sample size. Third, some factors that have been proven to affect GGT levels (such as sex, health status, and concurrent medication) were not considered in this study; therefore, residual confounding factors may have affected our results. Finally, this analysis is based on data from routine occupational health examinations; most participants are employees of enterprises and public institutions. There are differences in the sociological characteristics between those who undergo long-term physical examinations and those who rarely undergo physical examinations. Extrapolation of such results remains to be verified.

\section{Conclusion}

Taken together, this study demonstrated a synergistic effect of smoking and drinking on GGT levels, and established a dose-response relationship between smoking, alcohol consumption, and GGT levels. In real life, the exposures to smoking and drinking often coexist in the general population, and these exposures are avoidable risk factors. Therefore, our study findings are relevant for public health issues. In particular, our study emphasizes the importance of supervision over the ubiquitous and tedious daily tasks involved in health service work and encourages the public to adopt a non-smoking and nondrinking lifestyle to maintain their health. Further studies can include additional proven and unproven factors to observe the combined adverse impact of such factors on various liver enzymes and multiple health outcomes. Finally, further cohort studies should be conducted to obtain stronger evidence.

\section{Data Sharing Statement}

The data used and/or analyzed during the study are available from the corresponding author on reasonable request.

\section{Ethics Approval and Consent to Participate}

The present study was conducted in accordance with the Declaration of Helsinki guidelines, and the participants signed informed consent forms before being questioned and physical examined according to a protocol approved by the Ethics Committee of the First Affiliated Hospital of Xi'an Jiaotong University (No. KYLLSL-2020-156).

\section{Acknowledgments}

We would like to thank all participants in the study, all the staffs who coordinate the field work and all the investigators who contributed to data collection. This study was funded by the Key R\&D Program of Shaanxi under grant number 2020SF-071 and the research fund to YB from the University of Macau under grant number MYRG201900044-ICMS. The funders had no role in research design, data collection and analysis, and decision to submit the manuscript for publication.

\section{Disclosure}

The authors report no conflicts of interest in this work.

\section{References}

1. Whitfield JB. Gamma glutamyl transferase. Crit Rev Clin Lab Sci. 2001;38(4):263-355. doi:10.1080/20014091084227

2. Lee D, Kang H, Kim Y. Association between cigarette smoking and serum gam-ma-glutamyl transferase level. Respir Pulmonary Med. 2019;6:125.

3. Mohapatra E, Priya R, Nanda R, Patel S. Serum GGT and serum ferritin as early markers for metabolic syndrome. J Family Med Primary Care. 2020;9(7):3458-3463. doi:10.4103/jfmpc. jfmpc_570_20

4. Ndrepepa G, Kastrati A. Gamma-glutamyl transferase and cardiovascular disease. Ann Translat Med. 2016;4(24):481. doi:10.21037/ atm.2016.12.27

5. Mok Y, Son DK, Yun YD, Jee SH, Samet JM. $\gamma$-Glutamyltransferase and cancer risk: the Korean cancer prevention study. Int $j$ Cancer. 2016;138(2):311-319. doi:10.1002/ijc.29659

6. Nano J, Muka T, Ligthart S, et al. Gamma-glutamyltransferase levels, prediabetes and type 2 diabetes: a Mendelian randomization study. International Journal of Epidemiology. 2017;46(5):1400-1409. doi:10.1093/ije/dyx006 
7. Kim HW, Lee SH, Lee DH. Relationship of serum gamma-glutamyltransferase levels with pulmonary function and chronic obstructive pulmonary disease. Lung. 2014;192(5):719-727. doi:10.1007/s00408-014-9616-3

8. Mokdad AH, Marks JS, Stroup DF, Gerberding JL. Actual causes of death in the United States, 2000. JAMA. 2004;291(10):1238-1245. doi:10.1001/jama.291.10.1238

9. Mellinger J, Winder GS, Fernandez AC. HEP-20-1087: measuring the Alcohol in Alcohol-related Liver Disease: choices and Challenges for Clinical Research. Hepatology. 2020. doi:10.1002/hep.31539

10. Nivukoski U, Niemelä M, Bloigu A, et al. Impacts of unfavourable lifestyle factors on biomarkers of liver function, inflammation and lipid status. PLoS One. 2019;14(6):e0218463. doi:10.1371/journal. pone. 0218463

11. Avti PK, Kumar S, Pathak CM, Vaiphei K, Khanduja KL. Smokeless tobacco impairs the antioxidant defense in liver, lung, and kidney of rats. Toxicol Sci. 2006;89(2):547-553. doi:10.1093/toxsci/kfj041

12. Wannamethee SG, Shaper AG. Cigarette smoking and serum liver enzymes: the role of alcohol and inflammation. Ann Clin Biochem. 2010;47(Pt 4):321-326. doi:10.1258/acb.2010.009303

13. Jang ES, Jeong S-H, Hwang SH, et al. Effects of coffee, smoking, and alcohol on liver function tests: a comprehensive cross-sectional study. BMC Gastroenterology. 2012;12(1):145. doi:10.1186/1471230X-12-145

14. Satlin MJ, Lewis JS, Weinstein MP, et al. Clinical and Laboratory Standards Institute and European Committee on Antimicrobial Susceptibility Testing Position Statements on Polymyxin B and Colistin Clinical Breakpoints. Clin Infect Dis. 2020;71(9):e523e529. doi:10.1093/cid/ciaa121

15. Cochrane J, Chen H, Conigrave KM, Hao W. Alcohol use in China. Alcohol Alcoholism. 2003;38(6):537-542. doi:10.1093/alcalc/agg111

16. Wang J, Zhang D, Huang R, Li X, Huang W. Gamma-glutamyltransferase and risk of cardiovascular mortality: a dose-response meta-analysis of prospective cohort studies. PLoS One. 2017;12(2): e0172631. doi:10.1371/journal.pone.0172631

17. Franzini M, Scataglini I, Ricchiuti A, et al. Association between plasma gamma-glutamyltransferase fractions and metabolic syndrome among hypertensive patients. Sci Rep. 2017;7(1):1-8.

18. Abbas SH, Hussein ZA. Study Effect of Cigarette Smoking on the Liverenzymes. Int J Pharm Res. 2019;11(4).

19. Csordas A, Bernhard D. The biology behind the atherothrombotic effects of cigarette smoke. Nat Rev Cardiol. 2013;10(4):219-230. doi:10.1038/nrcardio.2013.8

20. Park EY, Lim MK, Oh JK, et al. Independent and supra-additive effects of alcohol consumption, cigarette smoking, and metabolic syndrome on the elevation of serum liver enzyme levels. PLoS One. 2013;8(5):e63439. doi:10.1371/journal.pone.0063439
21. Breitling LP, Raum E, Müller H, Rothenbacher D, Brenner H. Synergism between smoking and alcohol consumption with respect to serum gamma-glutamyltransferase. Hepatology. 2009;49 (3):802-808. doi:10.1002/hep.22727

22. Breitling LP, Arndt V, Drath C, Brenner H. Liver enzymes: interaction analysis of smoking with alcohol consumption or BMI, comparing AST and ALT to $\gamma$-GT. PLoS One. 2011;6(11):e27951. doi:10.1371/journal.pone.0027951

23. Coku V, Shkembi X. Serum Gamma-glutamyltransferase and Obesity: is there a Link? Med Arch. 2018;72(2):112-115. doi:10.5455/medarh.2017.72.112-115

24. Lee DH, Blomhoff R, Jacobs DR. Is serum gamma glutamyltransferase a marker of oxidative stress? Free Radic Res. 2004;38 (6):535-539. doi:10.1080/10715760410001694026

25. Breitling LP, Arndt V, Drath C, Rothenbacher D, Brenner $\mathrm{H}$. Smoking and $\gamma$-glutamyltransferase: opposite interactions with alcohol consumption and body mass index. PLoS One. 2010;5(9):e13116. doi:10.1371/journal.pone.0013116

26. Niemelä O, Nivukoski U, Bloigu A, Bloigu R, Aalto $M$, Laatikainen T. Laboratory test based assessment of WHO alcohol risk drinking levels. Scand J Clin Lab Invest. 2019;79(1-2):58-64. doi:10.1080/00365513.2019.1571625

27. Niemelä O, Niemelä M, Bloigu R, Aalto M, Laatikainen T. Where should the safe limits of alcohol consumption stand in light of liver enzyme abnormalities in alcohol consumers? PLoS One. 2017;12 (12):e0188574. doi:10.1371/journal.pone.0188574

28. Di Castelnuovo A, Costanzo S, Bagnardi V, Donati MB, Iacoviello L, de Gaetano G. Alcohol dosing and total mortality in men and women: an updated meta-analysis of 34 prospective studies. Arch Intern Med. 2006;166(22):2437-2445. doi:10.1001/archinte.166.22.2437

29. Agarwal S, Fulgoni VL, Lieberman HR. Assessing alcohol intake \& its dose-dependent effects on liver enzymes by 24-h recall and questionnaire using NHANES 2001-2010 data. Nutr J. 2016;15(1):62. doi:10.1186/s12937-016-0180-y

30. Stewart SH, Connors GJ, Hutson A. Ethnicity and gamma-glutamyltransferase in men and women with alcohol use disorders. Alcohol Alcoholism. 2007;42(1):24-27. doi:10.1093/ alcalc/ag1090

31. Whitehead TP, Robinson D, Allaway SL. The effects of cigarette smoking and alcohol consumption on serum liver enzyme activities: a dose-related study in men. Ann Clin Biochem. 1996;33(Pt 6):530-535. doi:10.1177/000456329603300607
International Journal of General Medicine

\section{Publish your work in this journal}

The International Journal of General Medicine is an international, peer-reviewed open-access journal that focuses on general and internal medicine, pathogenesis, epidemiology, diagnosis, monitoring and treatment protocols. The journal is characterized by the rapid reporting of reviews, original research and clinical studies across all disease areas. The manuscript management system is completely online and includes a very quick and fair peer-review system, which is all easy to use. Visit http://www.dovepress.com/ testimonials.php to read real quotes from published authors. 